\title{
An Ultrasound Phantom for Stenosing Flexor Tenosynovitis
}

Authors:

Joseph Gartrell Willis, ${ }^{1}$ James Barrett Harris," Jordan Austin George, ${ }^{1}$ Alvin Lee Day, ${ }^{2}$ David Resuehr ${ }^{1}$

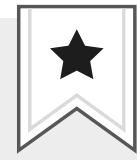

1. School of Medicine, University of Alabama at Birmingham, Birmingham, Alabama, USA

2. Division of Rheumatology, School of Medicine, University of South Carolina, Columbia, South Carolina, USA

*Correspondence to resuehr@uab.edu

Disclosure: $\quad$ The authors have declared no conflicts of interest.

Received: $\quad 09.10 .20$

Accepted: $\quad 10.02 .21$

Keywords: Medical education, musculoskeletal ultrasound (MSUS), stenosing flexor tenosynovitis, trigger finger, ultrasound simulation.

Citation:

EMJ Radiol. 2021;2[1]:58-64.

\section{INTRODUCTION}

Stenosing flexor tenosynovitis, often referred to as a trigger finger or trigger digit, is among the most common causes of hand pain in adults and often presents as a painful clicking or locking of a finger or thumb with movement. ${ }^{1}$ Trigger finger most often occurs in adults between the fourth and sixth decade of life and is more common in females than males. ${ }^{2}$ It can also be seen in children, typically prior to 8 years of age.

In the absence of pathology, flexion of the digits occurs smoothly as the flexor tendon glides within a system of annular and cruciform pulley sheaths. The pulley system prevents separation of the flexor tendon from the bone. Chronic repetitive friction is thought to cause most cases of primary idiopathic trigger finger by leading to thickening of the pulley sheath.1,3 The first annular (A1) pulley is most affected, and this is due to its receipt of the greatest degree of force during digital flexion. ${ }^{4}$

Trigger finger is considered a clinical diagnosis, but ultrasound assessment has been described as a useful adjunct in both diagnosis and treatment. ${ }^{5}$ Treatment for a trigger finger depends on severity and duration of symptoms.
Noninvasive options include a combination of nonsteroidal anti-inflammatory drugs, heat, ice, massage, and splinting. ${ }^{2}$ If noninvasive treatment fails, a corticosteroid injection (CSI) of the tendon sheath is suggested prior to consideration of surgical release of the pulley (Figure 1A and 1B). A single CSI was shown to provide up to 10 years of relief in $56 \%$ of female patients who presented for the first time with a trigger finger. ${ }^{6}$

Typically, a landmark-based CSI is performed, but a prospective study showed that only $37 \%$ of patients received all the injection within the sheath and $17 \%$ received no medication within the sheath. ${ }^{7}$ Ultrasound guidance has also been used and is considered the most accurate method. ${ }^{8-10}$ The efficacy of ultrasound-guided A1 pulley CSI was evaluated in a prospective study of 50 consecutive trigger fingers from 24 patients. $^{7}$ The patients who received the ultrasound-guided CSI showed complete resolution in $94 \%$ of fingers at 6 months. There are no large, blinded, randomised studies comparing the efficacy of ultrasound-guided to landmark-based injections in trigger fingers; however, a smaller, unblinded, randomised trial showed no difference in efficacy between ultrasound-guided and landmark-based CSI of trigger fingers. ${ }^{10}$ 


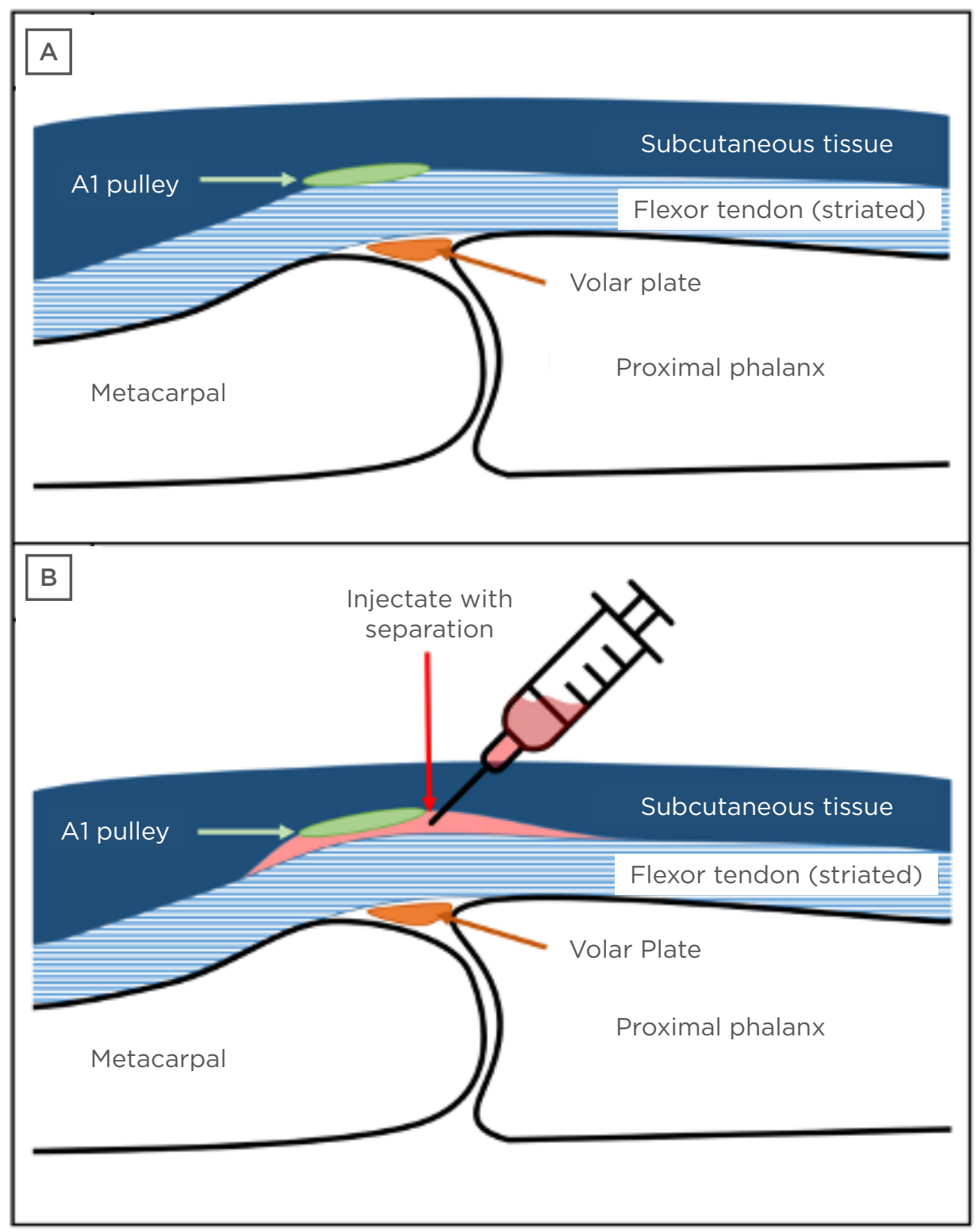

Figure 1: Schematic of the metacarpophalangeal joint with A) surrounding soft tissue structures and B) separation after the injection.

A1: first annular.

Ultrasound procedural phantoms have been developed to supplement education and allow for training prior to attempting a procedure on a patient.11-13 Simulation trainers used within a standardised curriculum of procedural instruction have also been shown to significantly improve participants' medical knowledge and technical skills. ${ }^{14}$ Commercial ultrasound training phantom models may be prohibitively expensive; however, low-cost homemade and three-dimensional (3D) printed models have more recently been developed. ${ }^{12,15}$ The authors herein propose a 3D-printed anatomical finger model embedded in ballistic gelatin as a low-cost ultrasound training phantom for procedural guidance of trigger finger injection.

\section{MATERIALS AND METHODS}

The finger models were created using a computeraided design software (Autodesk Fusion 360 Student Edition, Autodesk, San Rafael, California, USA), a Creality Ender 3 Pro Fused Deposition Modeling (FDM) 3D printer (Creality, Shenzhen, China), ballistic gelatin, and household materials. 
1. The distal, middle, proximal phalanx, and metacarpal bones were obtained through the database Thingiverse. The models were initially in the 'OBJ' format, which had to be converted to a solid model using the 'Mesh to BRep' command in Fusion 360 to allow the models to be edited prior to printing. The various models of the bones were then arranged and scaled appropriately in Fusion 360.

2. The bone model was scaled to the appropriate size within Fusion 360 according to a standard human finger.

3. After scaling, the hand was reduced to contain only the proximal phalanx and a portion of the distal metacarpal using the 'Cut' command in Fusion 360. The finger bone model was placed into a scaffold piece (Figure 2A), which allowed proper orientation for scanning and provided a method for attaching the tendon to the model via small holes.

4. A rectangular box model was created to allow for pouring of ballistic gelatin around the model (Figure 2B).

5. The scaffold and mould were modelled with features to create the proper thickness of the finger model (Figure 2C). The scaffold itself contained a ridge that indicated the intended height of the tendon, while the top of the mould indicated the thickness of the subcutaneous tissue in the average finger.

6. The mould and finger models were printed using the Creality Ender 3 Pro FDM printer using a $0.16 \mathrm{~mm}$ layer height with $0 \%$ infill (hollow) and 2 perimeters using SUNLU PLA Plus $1.75 \mathrm{~mm}$ 3D printer filament (Sunlu, Zhuhai City, China) with stock printer settings. Both models were sliced using PrusaSlicer to generate the file, or 'gcode', that allowed the 3D printer to print the model. These settings were chosen after various attempts with other infill settings in combination with previous literature recommendations. ${ }^{16}$

7. Flexor digitorum tendon models were assembled by wrapping Stren Magnathin Monofilament fishing line (Pure Fishing, Columbia, South Carolina, USA) around the finger bone model until a thickness of approximately 5 $\mathrm{mm}$ was achieved (Figure $2 \mathrm{C}$ ). The monofilament fishing line was made of nylon, had a $4 \mathrm{lb}$ test strength, and a $0.17 \mathrm{~mm}$ diameter. The line was tied into the model with holes for tight wrapping. The bundled strands were used to resemble the striated appearance of the flexor digitorum profundus and superficialis tendons (Figure 2D).

8. Ballistic gelatin was prepared as previously described ${ }^{17}$ with the following modifications: $1.5 \mathrm{~g}$ of gold mica powder was added to the dissolved ballistic gelatin prior to pouring to increase echogenicity.

9. The 3D-printed model was then attached to the tendon model. A thin layer of ballistic gelatin without mica powder was added onto the tendon above the metacarpophalangeal joint to resemble the $A 1$ pulley.

10. The finger model was placed into the 3D-printed rectangular mould and liquefied ballistic gelatin was poured into the mould to a level just above the tendon, covering the entire tendon from all sides (Figure 2E).

11. A second mixture of gelatin was created and poured into a separate rectangular mould to a height of $0.6 \mathrm{~cm}$ to serve as the subcutaneous tissue for the model. This gelatin was prepared by mixing $1.5 \mathrm{~g}$ of modelling clay into $25 \mathrm{~mL}$ of ballistic gelatin.

12. Once cooled and solidified, the hyperechoic gelatin layer was added to the ventral surface of the 3D-printed finger model (Figure 2F). Heated ballistic gelatin was applied on the edges of the gelatin layer to seal the perimeter of the two gelatin layers in the model. This created a potential space between the two layers to serve as the reservoir for the injectate.

13. Ultrasound transmission gel was used as the injectate due to its greater viscosity compared to water.

14. A 22 Gauge needle with a length of 1 inch was used for the simulated procedure.

Ultrasound images of the phantom were captured with a GE M12L linear transducer on a GE Venue 40 system (GE Healthcare, Chicago, Illinois, USA). The ultrasound machine was set to the 'Musculoskeletal (MSK)' preset and scans of the phantom were captured during an in-plane, long-axis injection. 


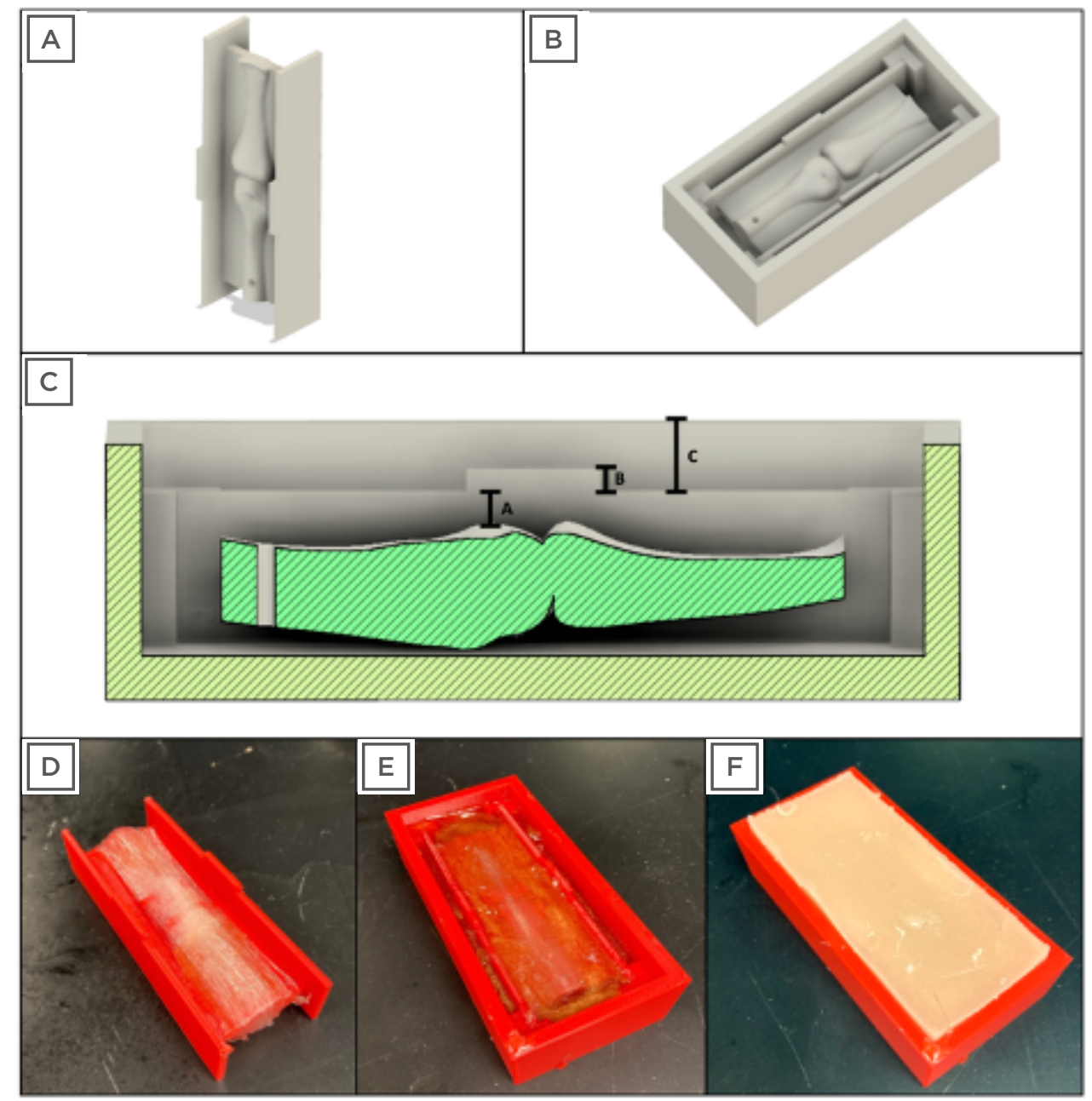

Figure 2: Computer-aided design and 3D-printed model of ultrasound phantom.

A) Render of the finger model placed into the scaffold prior to adding the tendon; B) render of the scaffold seated inside of the rectangular mould; C) cross-section of the finger model with key thicknesses indicated: A: thickness of the tendon between the bone and the subcutaneous tissue; B: thickness of and location of injectate; C: thickness of subcutaneous tissue; D) 3D-printed finger model with tendon made of fishing line; E) 3D-printed finger model after pouring of initial ballistic gelatin; F) layer of ballistic gelatin and modelling clay mixture layered on top of the initial 3D-printed finger model.

3D: three dimensional.

\section{RESULTS}

The 3D-printed finger phantom appeared anatomically and sonographically similar to images observed in a human finger both pre- and post-injection (Figure 3).

The pre-injection images had the following anatomical landmarks: metacarpal, proximal phalanx, volar plate, flexor digitorum complex (which included the tendons of flexor digitorum superficialis and flexor digitorum profundus), subcutaneous tissue, and the A1 pulley.
Additionally, the depth of each anatomical landmark was similar across all images (Figure 3E and 3F). The fishing line was able to create recognisable striations similar to the fibrillations in a human tendon. Additionally, the A1 pulley was visible in the phantom model, although slightly more hyperechoic than seen in a human finger.

Injection and separation were achieved, which resulted in a sonographic image similar to that of a human finger (Figure 3E and 3F). The separation was evident (Figure 3D), which indicated a successful injection. 


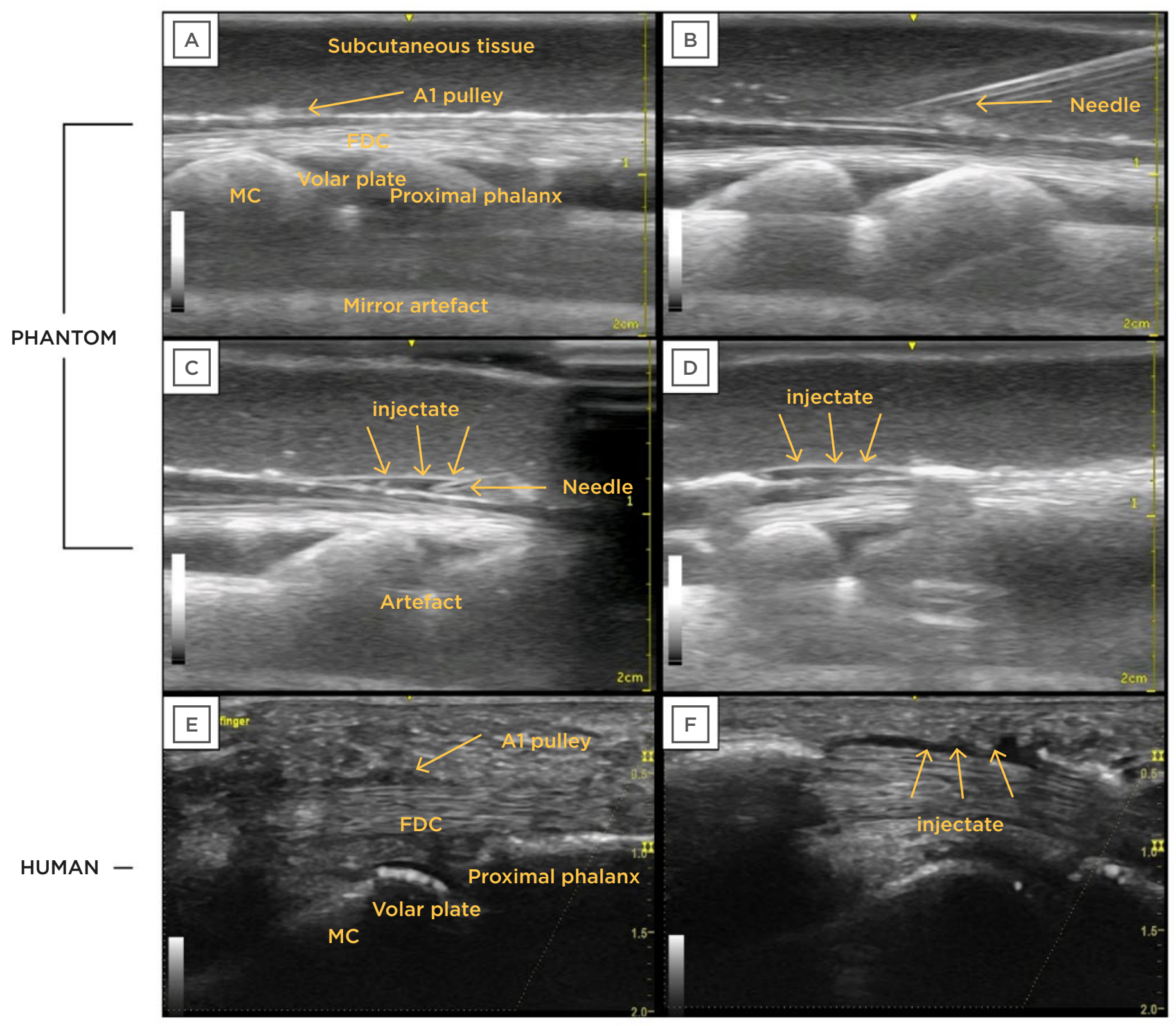

Figure 3: Pre- and post-injection ultrasound images of the 3D-printed phantom compared to a human finger, with all relevant anatomical features labelled.

A) Finger phantom with all relevant labels; B) needle approaching injection location; C) injection into location; D) phantom model post-injection with injectate and separation; E) human model pre-injection; F) human model postinjection with injectate and separation.

A1: first annular; FDC: flexor digitorum communis; MC: metacarpal.

\section{DISCUSSION}

The major strengths of the phantom were its sonographic similarity to a human finger, low cost, and relative ease of preparation.

For an ultrasound phantom to improve the skills of its trainees, it must mimic its human counterpart in its anatomy and echogenic appearance. By creating a finger model using computer-aided design software and 3D printing methods, the dimensions of the bony components were precise, with limited undesirable artifacts that could have been created using other construction methods.

Imaging of the metacarpophalangeal joint in long-axis showed soft tissue and bony landmarks sonographically similar to those in a 
human finger. The in-plane, long-axis injection technique was comparable to that often used in patients. The visual-spatial and tactile awareness required to perform the injection under ultrasound guidance was also similar between the human and phantom. The authors were unaware of any commercially available trigger finger models. The cost of the 3D-printed model presented in this study was less than \$20 USD.

Many universities have 3D printers available for use, which provides an accessible means of production. Additionally, all software was open-source or free to use under educational licensing agreements. Because the only area of the phantom that could be punctured was the ballistic gelatin outer portion, the 3D-printed bones and soft tissue components remained intact. Repeated needle insertions and injections created faint needle tract tracings after several injections, and the use of a hair dryer resolved the needle tracts. The PLA-printed finger bones in this setting have a near-unlimited lifespan and can be reused indefinitely.

\section{LIMITATIONS OF THE THREE-DIMENSIONAL-PRINTED FINGER PHANTOM}

While this study showed that a sonographically similar finger phantom model can be created using 3D printing, the experiment did have some noteworthy limitations. The final model created in this study was not physically similar to a human finger as the model was rectangular in shape and embedded within a mould. The authors believe that the rectangular model can be altered in a way that more closely resembles a finger, but the ability to create a potential space within such a finger prevented physical similarity. Further, given the shape of the mould, out-ofplane or transverse injection techniques were not performed.

As this project was carried out in a medical school environment, the ultrasound transducer selection was limited to a linear $12 \mathrm{MHz}$ transducer. Compact linear probes are often used for small-joint imaging and injections, and they typically have higher frequencies with improved near-field resolution. The overall sonographic appearance was unlikely to be affected by these differences, however.

As expected, the use of multiple materials created artefacts. The artefacts were likely reflections of the ultrasound waves between the hollow layers of the model. Scattering due to the mica powder was also noted. Ultrasound gel was used as the injectate due to the need for increased viscosity for tissue separation. Lower-viscosity fluids travelled back through the needle puncture site, which resulted in the fluid ejecting from the model. Fortunately, the ultrasound gel was anechoic and similar in sonographic appearance to a liquid injectate.

\section{CONCLUSION}

Ultrasound phantoms are a valuable training tool for both novices and experienced sonographers because of their nearly limitless versatility and, if home-made, low cost. The use of phantoms also supplements ultrasound education programmes. This manuscript describes a versatile and rather simple method for producing a low-cost, reusable finger phantom. Further studies are needed to validate the phantom model.

\section{References}

1. Ryzewicz M, Wolf JM. Trigger digits: principles, management, and complications. J Hand Surg Am. 2006;31(1):135-46.

2. Matthews A et al. Trigger finger: an overview of the treatment options. JAAPA. 2019;32(1):17-21

3. Hueston, JT, Wilson WF. The aetiology of trigger finger explained on the basis of intratendinous architecture. Hand. 1972;4(3):257-60.
4. Jeanmonod R et al. "Trigger Finger," StatPearls (2020), Treasure Island: StatPearls Publishing LLC.

5. Bianchi S et al. Ultrasound features of trigger finger: review of the literature. J Ultrasound Med. 2019;38(12):314154.

6. Wojahn RD et al. Long-term outcomes following a single corticosteroid injection for trigger finger. J Bone Joint Surg Am.
2014;96(22):1849-54.

7. Bodor M, Flossman T. Ultrasoundguided first annular pulley injection for trigger finger. J Ultrasound Med. 2009;28(6):737-43.

8. Orlandi D et al. Ultrasound-guided procedures around the wrist and hand: how to do. Eur J Radiol, 2014;83(7):1231-8.

9. Godey SK et al. A technique for accurate and safe injection of steroid 
in trigger digits using ultrasound guidance. Acta Orthop Belg. 2006;72(5):633-4

10. Cecen GS et al. Corticosteroid injection for trigger finger: blinded or ultrasound-guided injection? Arch Orthop Trauma Surg. 2015;135(1):125-31.

11. Yoo MC et al. Basic ultrasound curriculum for medical students: validation of content and phantom. $\mathrm{J}$ Laparoendosc Adv Surg Tech A. 2004;14(6):374-9

12. Day $A L$ et al. A versatile, low-cost, three-dimensional-printed ultrasound procedural training phantom of the human knee. EMJ Rheumatol. 2019;4(3):68-72

13. Oh EJ et al. Simulation-based training using a vessel phantom effectively improved first attempt success and dynamic needle-tip positioning ability for ultrasound-guided radial artery cannulation in real patients: an assessor-blinded randomized controlled study. PLoS One, 2020;15(6):e0234567.

14. Lenchus $\mathrm{J}$ et al. A blended approach to invasive bedside procedural instruction. Med Teach, 2011;33(2):11623.
15. Morrow DS et al. Versatile, reusable, and inexpensive ultrasound phantom procedural trainers. J Ultrasound Med. 2016;35(4):831-41.

16. Beaulieu A et al. Various 3D printed materials mimic bone ultrasonographically: 3D printed models of the equine cervical articular process joints as a simulator for ultrasound guided intra-articular injections. PLoS One. 2019;14(8):e0220332.

17. Burleson SL et al. A 1-week intensive ultrasound course for second-year medical students. Med Sci Educ. 2018;28(3):465-7. 